\title{
Toward a clean sample of ultra-luminous X-ray sources
}

\author{
M. López-Corredoira and C. M. Gutiérrez
}

\author{
Instituto de Astrofísica de Canarias, C/.vía Láctea, s/n, 38200 La Laguna (S/C de Tenerife), Spain \\ e-mail: martinlc@iac.es
}

Received 19 December 2005 / Accepted 21 February 2006

\begin{abstract}
Context. Observational follow-up programmes for the characterization of ultra-luminous X-ray sources (ULXs) require the construction of clean samples of such sources in which the contamination by foreground/background sources is minimum.

Aims. We calculate the degree of foreground/background contaminants among the ULX sample candidates in a published catalogue and compare these computations with available spectroscopic identifications.

Methods. We use statistics based on known densities of X-ray sources and AGN/QSOs selected in the optical. The analysis is done individually for each parent galaxy. The existing identifications of the optical counterparts are compiled from the literature.

Results. More than a half of the ULXs, within twice the distance of the major axis of the $25 \mathrm{mag} / \operatorname{arcsec}^{2}$ isophote from RC 3 nearby galaxies and with X-ray luminosities $L_{\mathrm{X}}[2-10 \mathrm{keV}] \geq 10^{39} \mathrm{erg} / \mathrm{s}$, are expected to be high redshift background QSOs. A list of $25 \mathrm{ob}-$ jects (clean sample) confirmed to be real ULXs or to have a low probability of being contaminant foreground/background objects is provided.
\end{abstract}

Key words. galaxies: statistics - galaxies: quasars: general

\section{Introduction}

ULXs (ultraluminous X-ray sources) are objects associated with some nearby galaxies, and which emit in X-ray with luminosities above the Eddington limit (so they cannot be stellar-mass X-ray binaries), but which are well below the typical emission in the center of AGNs. The physical nature of ULXs still is unclear. One of the most interesting possibilities is that they are black holes of intermediate masses (IMBHs; Colbert \& Mushotzky 1999; Makishima et al. 2000). Follow-up programmes over wide spectral ranges are essential to reveal the nature of these sources.

There is strong observational evidence that points to the existence of other kinds of objects linked to the parent galaxies: short-lived massive stars, usually in HII regions (e.g. Pakull \& Mirioni 2002; Gao et al. 2003; Kaaret et al. 2004; Zampieri et al. 2004; Liu et al. 2004; Smith et al. 2005; Lehmann et al. 2005), tentatively associated with intermediate-mass black holes. The ULX phenomenon is indeed closely connected to star formation activities, since ULXs preferentially occur in spiral arms of late-type or starbursts rather than in early-type galaxies (Kilgard et al. 2002; Schwartz et al. 2004; Liu et al. 2006). Actually, $70 \%$ of starburst galaxies host at least one ULX. However, as has been shown in the identification of several optical counterparts of ULXs (Lira et al. 2000; Arp et al. 2004; Gutiérrez \& López-Corredoira 2005, 2006; Gutiérrez 2006; Wong et al. 2006; Clark et al. 2005; e.g., IXO 1, 2 in NGC 720; see Table 1) the degree of contamination by foreground/background objects is large. The contamination is not total, so it is incorrect to think that all ULXs are QSOs with different redshifts (Burbidge et al. 2003).

Several statistical analyses (e.g. Ptak \& Colbert 2004; Schwartz et al. 2004; Irwin et al. 2004) of current compilations of ULXs confirm the relevance of such contamination. In this paper we address the problem of such a contamination in existing samples of ULXs, extending the work by these authors.
We analyse the catalogue by Colbert \& Ptak (2002) of ULX candidates and estimate the contamination in each galaxy and the most likely truly ULX sources. The paper is organized as follows: Sect. 2 describes the catalogue used and the corrections applied to obtain a homogeneous sample; the distribution of background sources and the method of computing probabilities are considered in Sects. 3 and 4; Sect. 5 presents the results of the statistical analysis; Sects. 6 and 7 present the discussion and conclusions, analysing also the possibility of some non-cosmological redshifts.

\section{Catalogues of ULXs and sample selection}

There are several catalogues of ULXs (ultraluminous X-ray sources), also called IXOs (intermediate-luminosity X-ray objects), built from ROSAT, XMM or Chandra data. Colbert \& Ptak (2002, C02 hereafter), and Liu \& Bregman (2005) catalogues are based in ROSAT data, while Schwartz et al. (2004) used Chandra data, and Liu \& Mirabel (2005) made a compilation of the literature values with a mixture of different data from different surveys, including data from Chandra and XMM X-ray telescopes. We analyse the $\mathrm{C} 02$ catalogue because i) a large fraction of the sources have been identified in the optical, and this allows a direct comparison with our statistical results; ii) the objects have been selected using data from just one X-ray instrument, providing a homogeneous sample; and iii) a correction is possible that includes objects that were known to be background objects.

The analysis should not be carried out on the sample as a whole because we are dealing with a catalogue with constraints in luminosity rather than in flux for galaxies within a wide range of distances; we cannot perform a simple calculation such as multiplying the total covered area by the average density of QSOs up to a given magnitude, because the limiting magnitude is different for each galaxy. Analysing the galaxies individually will also enable us to identify the galaxies likely to 
Table 1. Column 1: RC3 galaxy. Column 2: number of IXO in the C02 catalog, or from Table 1 in A04 (named with Axx, and the number xx of the row in that table). Column 3: flux in the range from 0.5 to $2.0 \mathrm{keV}$ [units $10^{-14} \mathrm{erg} / \mathrm{s} / \mathrm{cm}^{2}$ ]. Column $4:$ average number of expected sources up to the distance of this source to the centre of the galaxy, and brighter than its flux in X-ray. Column 5: probability that all candidate ULXs associated with the galaxy are indeed background sources with the observed distribution of X-ray fluxes. Column 6: $B$ magnitude of the most likely optical counterpart from the NOMAD database; letter " $\mathrm{d}$ " indicates that it is a diffuse source or a point-like source embedded in a halo; "a" indicates that the source is in the spiral arm of the galaxy. Column 7: average number of expected sources up to the distance of this source from the centre of the galaxy and brighter than its $B$ magnitude. Column 8: probability that all candidate ULXs associated with the galaxy are indeed background sources with the observed distribution of $B$ magnitudes. Column 9: spectroscopic identification [reference]. References of the spectroscopical identification: 1: A04; 2: Zampieri et al. (2004); 3: Gutiérrez \& López-Corredoira (2006); 4: Gutiérrez (2006); 5: Lehmann et al. (2005); 6: Kaaret et al. (2004); 7: Gutiérrez \& López-Corredoira (2005); 8: Ramsey et al. (2006); 9: Liu et al. (2005); 10: NED database; 11: Liu et al. (2004); 12: Arp (1977); 13: Wong et al. (2006) (we call “QSO” those objects classified by Wong et al. (2006) as AGN, since most of these sources are presumably point-like QSOs rather than extended galaxies); 14: Ghosh et al. (2006b); 15: Pakull \& Mirioni (2002); 16: Mucciarelli et al. (2005); 17: Kaaret (2005).

\begin{tabular}{|c|c|c|c|c|c|c|c|c|}
\hline Galaxy & IXO & $S_{X, i}$ & $\lambda_{X, i}$ & $P_{\mathrm{X}}$ & $m_{B, i}$ & $\lambda_{B, i}$ & $P_{B}$ & Spectr. ident. \\
\hline \multirow[t]{2}{*}{ NGC 720} & 1 & 1.1 & 0.97 & $5 \times 10^{-2}$ & 20.1 & 0.16 & $7 \times 10^{-4}$ & QSO $[1,13]$ \\
\hline & 2 & 4.4 & $8.9 \times 10^{-2}$ & & 18.7 & $5.1 \times 10^{-3}$ & & QSO $[1,13]$ \\
\hline NGC 891 & 3 & 7.9 & 0.16 & 0.1 & $>21.5$ & $>1.46$ & $>0.8$ & \\
\hline NGC 1042 & 4 & 17 & $3.5 \times 10^{-3}$ & $3 \times 10^{-3}$ & $>21.5(a)$ & $>0.12$ & $>0.1$ & \\
\hline \multirow[t]{3}{*}{ NGC 1073} & 5 & 2.2 & 0.12 & $10^{-4}$ & 13.1(a) & $1.2 \times 10^{-7}$ & $8 \times 10^{-12}$ & HII [17] \\
\hline & A3 & 2.8 & $5.5 \times 10^{-2}$ & & $V=20.0$ & $2.4 \times 10^{-2}$ & & QSO [1] \\
\hline & A5 & 7.0 & $2.7 \times 10^{-2}$ & & $V=18.8$ & $4.5 \times 10^{-3}$ & & QSO [1] \\
\hline NGC 1291 & 6 & 9.8 & $5.8 \times 10^{-2}$ & $6 \times 10^{-2}$ & $>21.5$ & $>0.78$ & $>0.5$ & \\
\hline \multirow[t]{2}{*}{ NGC 1313} & 7 & 210 & $1.5 \times 10^{-5}$ & $6 \times 10^{-8}$ & $>21.5$ & $>3.2 \times 10^{-2}$ & $>6 \times 10^{-3}$ & HII $[15]$ \\
\hline & 8 & 84 & $3.8 \times 10^{-3}$ & & 20.0 & 0.47 & & O-BV or GI in HII $[2,8,15,16]$ \\
\hline \multirow[t]{6}{*}{ NGC 1316} & 9 & 8.0 & 0.59 & $2 \times 10^{-3}$ & 19.5 & 0.70 & $>3 \times 10^{-4}$ & QSO [13] \\
\hline & 10 & 4.0 & 0.27 & & 20.2 & 0.27 & & QSO [13] \\
\hline & 11 & 2.5 & 0.36 & & $>21.5$ & $>0.51$ & & \\
\hline & 12 & 2.0 & 1.04 & & $>21.5$ & $>1.01$ & & \\
\hline & 13 & 2.0 & 2.95 & & 21.2 & 2.33 & & \\
\hline & 14 & 2.5 & 3.05 & & 19.1 & 0.23 & & QSO [13] \\
\hline \multirow[t]{3}{*}{ NGC 1365} & 15 & 5.9 & 0.29 & $4 \times 10^{-2}$ & 19.4 & 0.17 & $10^{-3}$ & QSO [13] \\
\hline & 16 & 5.9 & 1.01 & & 18.7 & $2.7 \times 10^{-2}$ & & QSO [13] \\
\hline & A6 & 5.9 & 0.48 & & $V=19.7$ & 0.47 & & QSO [1] \\
\hline \multirow[t]{4}{*}{ NGC 1399} & 17 & 3.1 & $2.9 \times 10^{-2}$ & $10^{-7}$ & $>21.5$ & $>5.7 \times 10^{-2}$ & $>6 \times 10^{-9}$ & \\
\hline & 18 & 6.2 & $4.2 \times 10^{-3}$ & & $>21.5$ & $>2.6 \times 10^{-2}$ & & \\
\hline & 19 & 31 & $1.4 \times 10^{-2}$ & & 20.1 & 0.37 & & QSO [13] \\
\hline & 20 & 4.9 & 0.23 & & 15.2 & $4.3 \times 10^{-5}$ & & QSO [13] \\
\hline NGC 1427A & 21 & 5.0 & $4.3 \times 10^{-3}$ & $4 \times 10^{-3}$ & 18.4 & $2.9 \times 10^{-4}$ & $3 \times 10^{-4}$ & \\
\hline IC342 & 22 & 48 & $6.5 \times 10^{-3}$ & $6 \times 10^{-3}$ & 17.8 & $6.3 \times 10^{-3}$ & $6 \times 10^{-3}$ & HII $[3,8,15]$ \\
\hline NGC 1553 & 23 & 5.5 & 0.10 & 0.1 & 20.8 & 0.32 & 0.3 & \\
\hline NGC 1566 & 24 & 5.8 & $4.8 \times 10^{-3}$ & $5 \times 10^{-3}$ & $>21.5(a)$ & $>2.7 \times 10^{-2}$ & $>3 \times 10^{-2}$ & \\
\hline \multirow[t]{3}{*}{ NGC 1672} & 25 & 1.8 & 0.11 & $2 \times 10^{-5}$ & $>21.5(a)$ & $>9.8 \times 10^{-2}$ & $\sim 2 \times 10^{-14}$ & \\
\hline & 26 & 5.6 & $1.1 \times 10^{-2}$ & & $15.1(\mathrm{da})$ & $2.9 \times 10^{-4}$ & & \\
\hline & 27 & 4.5 & $2.5 \times 10^{-2}$ & & $\mathrm{R}=13.4(\mathrm{da})$ & $\sim 1.4 \times 10^{-7}$ & & \\
\hline NGC 1792 & 28 & 3.4 & $1.3 \times 10^{-2}$ & $10^{-2}$ & $>21.5$ & $>3.0 \times 10^{-2}$ & $>3 \times 10^{-2}$ & \\
\hline NGC 1961 & 29 & 5.3 & $7.2 \times 10^{-2}$ & $7 \times 10^{-2}$ & 20.6 & 0.17 & 0.2 & \\
\hline Mrk 3 & 30 & 4.0 & $4.2 \times 10^{-2}$ & $4 \times 10^{-2}$ & $>21.5$ & 0.13 & $>0.1$ & \\
\hline Holmberg II & 31 & 360 & $3.7 \times 10^{-5}$ & $4 \times 10^{-5}$ & 14.2 & $1.3 \times 10^{-6}$ & $10^{-6}$ & O4V-B3Ib in HII $[5,6,8,15]$ \\
\hline \multirow[t]{2}{*}{ NGC 2775} & 32 & 2.2 & 0.55 & $6 \times 10^{-2}$ & 18.1 & $5.7 \times 10^{-3}$ & $2 \times 10^{-4}$ & QSO $[4,13]$ \\
\hline & 33 & 4.4 & 0.16 & & 19.2 & $3.9 \times 10^{-2}$ & & QSO $[7,13]$ \\
\hline NGC 3031 & 34 & 360 & $1.4 \times 10^{-3}$ & $10^{-3}$ & 20.4 & 3.00 & 0.95 & 4-6 Myr blue stars in HII $[8,15]$ \\
\hline NGC 3226 & 35 & 2.3 & 0.22 & 0.2 & 18.4 & $4.3 \times 10^{-3}$ & $4 \times 10^{-3}$ & local M star $[4,13]$ \\
\hline NGC 3256 & 36 & 2.1 & 0.34 & 0.3 & $>21.5$ & $>0.36$ & $>0.3$ & \\
\hline IC 2597 & 37 & 23 & $3.0 \times 10^{-3}$ & $3 \times 10^{-3}$ & 20.8 & $9.5 \times 10^{-2}$ & 0.1 & Emis. line Gal. [4, 13] \\
\hline NGC 3310 & 38 & 4.1 & $1.6 \times 10^{-2}$ & $2 \times 10^{-2}$ & 17.5 & $1.5 \times 10^{-4}$ & $10^{-4}$ & \\
\hline \multirow[t]{5}{*}{ NGC 3628} & 39 & 24 & $3.8 \times 10^{-5}$ & $3 \times 10^{-10}$ & $>21.5$ & $>2.3 \times 10^{-3}$ & $>8 \times 10^{-8}$ & \\
\hline & A9 & 770 & $9.0 \times 10^{-2}$ & & $V=19.5$ & 0.10 & & QSO [1] \\
\hline & A10 & 15 & $4.9 \times 10^{-2}$ & & $V=19.2$ & $9.3 \times 10^{-2}$ & & QSO [1] \\
\hline & A13 & 9.7 & 0.22 & & $V=19.6$ & 0.42 & & QSO [1] \\
\hline & A14 & 31 & $7.8 \times 10^{-2}$ & & $V=18.3$ & $9.1 \times 10^{-2}$ & & QSO [1] \\
\hline NGC 3923 & 40 & 7.3 & 0.11 & 0.1 & 19.0 & $3.7 \times 10^{-2}$ & $4 \times 10^{-2}$ & QSO $[4,13]$ \\
\hline UGC 7009 & 41 & 8.2 & $1.4 \times 10^{-3}$ & $10^{-3}$ & $>21.5$ & $1.4 \times 10^{-2}$ & $10^{-2}$ & \\
\hline NGC 4088 & 42 & 5.0 & $2.7 \times 10^{-3}$ & $3 \times 10^{-3}$ & $14.8(\mathrm{da})$ & $2.4 \times 10^{-7}$ & $3 \times 10^{-7}$ & \\
\hline \multirow[t]{3}{*}{ NGC 4151} & 43 & 2.2 & 1.02 & $6 \times 10^{-4}$ & 18.8 & $2.6 \times 10^{-2}$ & $8 \times 10^{-4}$ & Emis.line Gal. $[3,12,13]$ \\
\hline & 44 & 1.1 & 0.46 & & 20.9 & 0.16 & & \\
\hline & A 15 & 62 & $3.8 \times 10^{-3}$ & & $V=20.3$ & 0.41 & & QSO [1] \\
\hline
\end{tabular}


Table 1. continued

\begin{tabular}{|c|c|c|c|c|c|c|c|c|}
\hline Galaxy & IXO & $S_{X, i}$ & $\lambda_{X, i}$ & $P_{\mathrm{X}}$ & $m_{B, i}$ & $\lambda_{B, i}$ & $P_{B}$ & Spectr. ident. \\
\hline NGC 4168 & A16 & 1.2 & $4.2 \times 10^{-2}$ & $4 \times 10^{-2}$ & $V=18.7$ & $4.2 \times 10^{-4}$ & $4 \times 10^{-4}$ & QSO [1] \\
\hline NGC 4203 & 45 & 2.2 & 0.29 & 0.3 & 18.3 & $4.3 \times 10^{-3}$ & $4 \times 10^{-3}$ & QSO [7] \\
\hline NGC 4254 & 46 & 14 & $6.2 \times 10^{-3}$ & $6 \times 10^{-3}$ & $>21.5$ & $>0.15$ & $>0.1$ & \\
\hline NGC 4319 & A21 & 360 & $4.3 \times 10^{-6}$ & $4 \times 10^{-6}$ & $V=15.2$ & $9.9 \times 10^{-7}$ & $10^{-6}$ & QSO [1] \\
\hline \multirow[t]{3}{*}{ NGC 4321} & 47 & 3.2 & 0.81 & 0.2 & 20.3 & 0.64 & $>0.1$ & \\
\hline & 48 & 1.6 & 2.41 & & $>21.5$ & $>1.92$ & & \\
\hline & 49 & 2.0 & 0.67 & & $>21.5$ & $>0.66$ & & \\
\hline \multirow[t]{3}{*}{ NGC 4374} & 50 & 16 & $2.0 \times 10^{-4}$ & $2 \times 10^{-7}$ & $>21.5$ & $>6.3 \times 10^{-3}$ & $>6 \times 10^{-6}$ & \\
\hline & 51 & 6.4 & 0.11 & & $>21.5$ & $>0.73$ & & \\
\hline & A22 & 10 & $1.8 \times 10^{-2}$ & & $V=18.5$ & $3.1 \times 10^{-3}$ & & QSO [1] \\
\hline NGC 4373 & 52 & 2.2 & 0.20 & 0.2 & 18.7 & $3.7 \times 10^{-3}$ & $4 \times 10^{-3}$ & QSO [13] \\
\hline NGC 4395 & 53 & 35 & $3.1 \times 10^{-3}$ & $3 \times 10^{-3}$ & $>21.5$ & $>0.36$ & $>0.3$ & \\
\hline NGC 4438 & 54 & 13 & $2.6 \times 10^{-2}$ & $3 \times 10^{-2}$ & 19.5 & $6.9 \times 10^{-2}$ & $7 \times 10^{-2}$ & QSO [3] \\
\hline \multirow[t]{7}{*}{ NGC 4472} & 55 & 2.0 & 3.44 & $10^{-4}$ & 20.8 & 2.03 & $>3 \times 10^{-5}$ & \\
\hline & 56 & 4.1 & 1.14 & & 20.8 & 2.13 & & \\
\hline & 57 & 10 & 0.21 & & 18.2 & $3.4 \times 10^{-2}$ & & QSO [3] \\
\hline & 58 & 4.1 & 0.96 & & 18.4 & $4.7 \times 10^{-2}$ & & QSO [7] \\
\hline & 59 & 3.2 & 2.19 & & 21.0 & 3.27 & & \\
\hline & 60 & 13 & $9.6 \times 10^{-2}$ & & 21.0 & 1.42 & & \\
\hline & 61 & 2.6 & 0.93 & & $>21.5$ & $>1.36$ & & \\
\hline NGC 4485 & 62 & 11 & $2.7 \times 10^{-4}$ & $3 \times 10^{-4}$ & $13.2(\mathrm{~d})$ & $4.4 \times 10^{-9}$ & $4 \times 10^{-9}$ & \\
\hline \multirow[t]{2}{*}{ NGC 4552} & 63 & 5.1 & 0.26 & $5 \times 10^{-2}$ & 20.4 & 0.48 & $5 \times 10^{-2}$ & \\
\hline & 64 & 3.2 & 0.25 & & 20.0 & 0.14 & & \\
\hline \multirow{2}{*}{ NGC 4559} & 65 & 39 & $1.5 \times 10^{-3}$ & $5 \times 10^{-8}$ & 12.6 & $7.0 \times 10^{-8}$ & $>10^{-10}$ & 4 blue/red superg. in HII $[3,9,15]$ \\
\hline & 66 & 24 & $3.4 \times 10^{-5}$ & & $>21.5$ & $>2.1 \times 10^{-3}$ & & \\
\hline NGC 4565 & 67 & 21 & $5.0 \times 10^{-4}$ & $5 \times 10^{-4}$ & $>21.5$ & $>2.5 \times 10^{-2}$ & $>2 \times 10^{-2}$ & \\
\hline NGC 4631 & 68 & 12 & $1.6 \times 10^{-2}$ & $2 \times 10^{-2}$ & $V=14.2(\mathrm{da})$ & $\sim 2.1 \times 10^{-6}$ & $\sim 2 \times 10^{-6}$ & HII [15] \\
\hline \multirow[t]{3}{*}{ NGC 4649} & 69 & 5.2 & $8.8 \times 10^{-2}$ & $4 \times 10^{-3}$ & 18.3 & $5.4 \times 10^{-3}$ & $4 \times 10^{-5}$ & QSO [7] \\
\hline & 70 & 4.1 & 0.71 & & 18.4 & $3.6 \times 10^{-2}$ & & \\
\hline & 71 & 10 & 0.16 & & 19.8 & 0.46 & & QSO [7] \\
\hline NGC 4698 & A 23 & 10 & $4.8 \times 10^{-3}$ & $5 \times 10^{-3}$ & $V=20.5$ & $3.0 \times 10^{-2}$ & $3 \times 10^{-2}$ & QSO [1] \\
\hline \multirow[t]{2}{*}{ NGC 4861} & 72 & 9.1 & $4.9 \times 10^{-3}$ & $9 \times 10^{-7}$ & $9.5(\mathrm{a})$ & $6.9 \times 10^{-11}$ & $>10^{-12}$ & HII $[10,15]$ \\
\hline & 73 & 29 & $1.9 \times 10^{-4}$ & & $>21.5$ & $>1.6 \times 10^{-2}$ & & HII [15] \\
\hline NGC 5055 & 74 & 35 & $1.3 \times 10^{-2}$ & $10^{-2}$ & 18.4 & $2.3 \times 10^{-2}$ & $2 \times 10^{-2}$ & \\
\hline \multirow[t]{2}{*}{ NGC 5128} & 75 & 38 & $9.2 \times 10^{-3}$ & $3 \times 10^{-6}$ & 10.7 & $1.3 \times 10^{-8}$ & $>4 \times 10^{-9}$ & local M star $[10,13]$ \\
\hline & 76 & 150 & $2.9 \times 10^{-4}$ & & $>21.5$ & $>0.36$ & & \\
\hline NGC 5204 & 77 & 63 & $1.3 \times 10^{-5}$ & $10^{-5}$ & $>21.5$ & $>3.8 \times 10^{-3}$ & $>4 \times 10^{-3}$ & B0Ib in nebula $[11,15]$ \\
\hline \multirow[t]{4}{*}{ NGC 5194} & 78 & 6.5 & $3.8 \times 10^{-2}$ & $2 \times 10^{-8}$ & $>21.5(a)$ & $>0.26$ & $>5 \times 10^{-13}$ & \\
\hline & 79 & 81 & $1.3 \times 10^{-2}$ & & $13.0(\mathrm{da})$ & $9.8 \times 10^{-8}$ & & \\
\hline & 80 & 16 & $8.1 \times 10^{-3}$ & & 16.9 (a) & $2.5 \times 10^{-4}$ & & young star cluster [9] \\
\hline & 81 & 13 & $1.4 \times 10^{-2}$ & & $>21.5(\mathrm{a})$ & $>0.29$ & & ? in young region [9] \\
\hline NGC 5236 & 82 & 33 & $9.8 \times 10^{-3}$ & $10^{-2}$ & $>21.5$ & $>0.98$ & $>0.6$ & \\
\hline NGC 5457 & 83 & 20 & 0.12 & 0.1 & $>21.5$ & $>4.99$ & $>0.99$ & \\
\hline NGC 5775 & 84 & 2.3 & 0.43 & 0.3 & 19.6 & $7.6 \times 10^{-2}$ & $7 \times 10^{-2}$ & QSO $[7,14]$ \\
\hline NGC 6946 & 85 & 15 & $1.9 \times 10^{-3}$ & $2 \times 10^{-3}$ & $>21.5(\mathrm{a})$ & $>5.2 \times 10^{-2}$ & $>5 \times 10^{-2}$ & \\
\hline NGC 7314 & 86 & 3.2 & $6.4 \times 10^{-2}$ & $6 \times 10^{-2}$ & 18.6 & $1.7 \times 10^{-3}$ & $>2 \times 10^{-3}$ & \\
\hline NGC 7590 & 87 & 6.4 & $1.3 \times 10^{-3}$ & $10^{-3}$ & $>21.5$ (a) & $>8.8 \times 10^{-3}$ & $>9 \times 10^{-3}$ & \\
\hline
\end{tabular}

be totally/partially contaminated and those that are not, and will provide a useful tool for ULX searches and optimizing future follow-up studies. Furthermore, we compile the recent literature about the observation of the optical counterparts of these candidate ULXs that will corroborate our statistical analysis or will show whether there is some possible anomalous excess of different redshift objects around the parent galaxies.

C02 presented a catalogue of 87 candidate ULXs, which were observed in X-ray with ROSAT HRI (Voges et al. 1999); they were around bright galaxies from the RC3 catalog whose velocity is less than $5000 \mathrm{~km} \mathrm{~s}^{-1}$ and have more than $90 \%$ of the galaxy in the field of view of the ROSAT HRI observations (the total number of galaxies within these constraints was 766). They were detected by simply measuring the luminosity of these ROSAT sources assuming they are at the distance of a parent galaxy that is nearby: their sample of IXOs follows the constraint that the ULX is at an angular distance less than $2 R_{25}$ from the centre of an RC3 galaxy, where $R_{25}$ is the major axis of the $25 \mathrm{mag} / \operatorname{arcsec}^{2}$ isophote, and the luminosity assuming the ULX is at the same distance as the galaxy $L_{\mathrm{X}}[2-10 \mathrm{keV}] \geq 10^{39} \mathrm{erg} / \mathrm{s}$. A total of 54 galaxies had at least one of the 87 candidate ULXs with these constraints, and some galaxies were surrounded by up to seven candidate ULXs.

C02 have not included in their catalogue some sources observed by ROSAT HRI that could be classified as candidate ULXs according to their constraints because they were known to be QSOs with a redshift different from that of the RC3 galaxy. Arp et al. (2004, hereafter A04) (Table 1) list these sources; there are 12 extra candidate ULXs in this list which follow the $\mathrm{C} 02$ constraints. Even if they are background sources that 
have been discarded as ULXs associated with the galaxy, they should be included in the catalogue if we wish to select a statistical sample. Indeed, as we will see throughout this paper, many of the candidate ULXs turned out later also to be background sources, so there is no reason to separate sources that were known to be background sources before or after the $\mathrm{C} 02$ compilation. If we include these sources, there are 99 candidate ULXs around 57 galaxies. Hereafter we will denote this sample as C02c ("Colbert \& Ptak 2002, corrected"). They are listed in Table 1.

\section{Distribution of background sources}

To estimate the contamination in the $\mathrm{C} 02 \mathrm{c}$ catalogue we consider the distribution of background sources in two different wavelength ranges: i) any source, $W \equiv X, \mathrm{X}$-ray at frequencies between 0.5 and $2.0 \mathrm{keV}$; ii) QSOs, $W \equiv B, B$ optical filter.

For the X-ray, we use the cumulative counts by Hasinger et al. (1993) obtained in the Lockman Hole from ROSAT-PSPC counts using the flux spectral shape they also derived. A parameterization of their Fig. 8 with two linear regimes gives (in units of $\mathrm{deg}^{-2}$ ) as a function of the flux $S_{X, i}$ (units: $\mathrm{erg} / \mathrm{s} / \mathrm{cm}^{2}$ ):

$N_{X, i}\left(>S_{i}\right)=\left\{\begin{array}{ll}1.0 \times 10^{-21} S_{X, i}^{-1.66}, & S_{X, i} \geq 2 \times 10^{-14} \\ 2.8 \times 10^{-12} S_{X, i}^{-0.97}, & S_{X, i}<2 \times 10^{-14}\end{array}\right\}$.

This law is valid in principle for the range $10^{-15}<S_{X, i}<10^{-12}$, where most of our ROSAT sources lie. Hasinger (1998) gives the counts for a wider regime, and the linear regimes are present in wider ranges, although the exponents change slightly for larger values of $S_{X, i}$. Zickgraf et al. (2003, Fig. 18) give counts for brighter X-ray sources. For the estimation of probabilities (see next section), the law of Eq. (1) is accurate enough.

We take the ROSAT X-ray flux of the sources, $S_{X, i}$, from $\mathrm{C} 02 \mathrm{c}$. They give the luminosity for the range $2 \mathrm{keV}<$ $v<10 \mathrm{keV}$ but this can be converted into fluxes if we divide the luminosity by $4 \pi d_{\text {galaxy }}^{2}$; and $S_{X, i} \equiv S_{i}[0.5 \mathrm{keV}<v<$ $2 \mathrm{keV}]=0.548 S_{i}[2 \mathrm{keV}<v<10 \mathrm{keV}]$. The factor 0.548 stems from the assumption that the $\mathrm{X}$-ray spectrum of the sources is $S[v] \mathrm{d} v \propto v^{-0.7} \mathrm{~d} v$, an assumption also adopted by $\mathrm{C} 02$ to calculate their luminosities.

For the estimation of the background QSOs in the $B$-band, we use the counts by Cheney \& Rowan-Robinson (1981) for $m_{B} \leq 18.5$ and Boyle et al. (2000) for $m_{B}>18.5$ (see the fit in Fig. A.1 of López-Corredoira \& Gutiérrez 2004) as a function of the photographic magnitude $m_{b_{j}, i}$. Where no $B$-magnitude is available for a candidate ULX optical counterpart, we use the $V$-magnitude (in QSOs, $(B-V)$ is small). Since we are only interested in a rough approximation of the counts, no colour correction for the difference between photographic and Johnson filter or with $V$ in case of a few QSOs from A04 is applied. Boyle et al. (2000) counts of colour-preselected QSOs agree more or less with the counts from a more complete spectroscopic survey by Meyer et al. (2001). The counts include both QSOs and Seyfert 1 galaxies (Seyfert 1-type galaxies would form $\sim 10 \%$ of the total counts) but not Seyfert 2, LINERs or other types of AGNs. According to Hao et al. (2005), Seyfert 2 galaxies would be less than four time more numerous than Seyfert 1, so the inclusion of Seyfert 2 would increase the counts up to $40 \%$. Most of our sources are presumably point-like QSOs rather than extended galaxies, given the images of the optical counterparts. Since we are interested in the order of magnitude, it is not so important whether an AGN is a QSO or a Seyfert galaxy.
The counts are (units $\operatorname{deg}^{-2}$ ):

$$
\begin{aligned}
& N_{B, i}\left(<m_{B, i}\right)= \\
& \left\{\begin{array}{ll}
10^{-2.8+0.8\left(m_{B, i}-15\right)}, & m_{B, i} \leq 18.5 \\
1981.0-214.2 m_{b_{j}, i}+5.792 m_{b_{j}, i}^{2}, & m_{B, i}>18.5
\end{array}\right\} .
\end{aligned}
$$

In this case, the $B$ magnitudes were taken from the NOMAD database $^{1}$ of the most likely optical counterpart (the brightest candidate closest to and within $10^{\prime \prime}$ of the X-ray source). The nominal positional accuracy of ROSAT HRI is 5 arcsec (Voges et al. 1999). If we compare the position of the 14 candidate ULXs common to CP02 (with ROSAT) and Schwartz et al. (2004) (with Chandra), we find that the average difference is 2.4 arcsec. If we do not find any counterpart within $10^{\prime \prime}$, we attribute this a magnitude $m_{B}>21.5$, i.e. higher than the limiting magnitude of the Palomar plates. It may happen that the optical source is a false counterpart because the real ULX is much fainter; however, the probability is not very high in regions away from the galaxy. In fact, the probability of observing an object with magnitude $\sim 20$ within a circle of radius $10^{\prime \prime}$ is $\leq 10^{-3}$ for QSOs (Boyle et al. 2000) and $\sim 10^{-2}$ for high galactic latitude stars (Robin et al. 2003), which we consider negligible. A higher contamination could be present in spiral arms, where the density of new-born stars in HII regions could be elevated. For instance, Kaaret (2005) shows that IXO 5 is much fainter than the magnitude of the brightest source in A04, found as an optical counterpart. In this case, and when spectroscopy of the optical counterpart has not revealed a QSO or stellar nature, the contamination with regard to the visible magnitude would be underestimated but never overestimated, so we will obtain a minimum of the ratio of contamination.

\section{Probability of being a background source}

From the catalogue of ULXs in Table 1 we calculate the probability that they are background objects (mainly background QSOs) instead of X-ray sources associated with their putative parent galaxies.

Given a galaxy with $n$ ULXs whose angular distances from its centre are $\alpha_{i}(\mathrm{deg})$ and background density $N_{W, i}\left(\mathrm{deg}^{-2}\right)$ of sources brighter than the flux/"apparent magnitude" of this source in a wavelength range $W$, the probability that all of them are background sources is:

$P_{W} \sim \mathrm{e}^{-\lambda_{W: \max }}\left(\sum_{i=n}^{\infty} \frac{\lambda_{W: \max }^{i}}{i !}\right)\left(\prod_{j=1, j \neq i i_{W: \max }}^{n} \frac{2 \lambda_{W, j}}{\lambda_{W: \max }}\right)$,

$\lambda_{W: \max }=\operatorname{Maximum}\left[\lambda_{W, i}\right] ; \quad i_{W: \max } / \lambda_{W, i_{\max }}=\lambda_{W: \max }$

$\lambda_{W, i}=\pi \alpha_{i}^{2} N_{W, i}$

The first two factors represent a Poissonian distribution for $n$ background sources equivalent to $i_{W: \max }$ (the fainter and more distant source). The third factor, $\left(\prod_{j=1, j \neq i_{W: \max }}^{n} 2 \lambda_{W, j} / \lambda_{W: \max }\right)$, takes into account that the sources might be brighter or closer to the centre of the galaxy than normally expected in a random process. Randomly, one would expect that $\lambda_{j} / \lambda_{\max } \approx 1 / 2$ on average, so this third factor should be nearly unity; but in some cases, some sources are much brighter than $i_{W: \max }$ or much closer to the centre than $i_{W: \max }$, and

\footnotetext{
${ }^{1}$ http://www . navy.mil/nomad.html
} 
in such cases the third factor would be much lower than one, reflecting this improbable difference in brightness or angular distance. For example, in the case of three candidate ULXs with magnitudes 20,16 and 15 and distances around 1 arcmin from the nucleus of the galaxy, we will calculate the Poissonian probability of having background QSOs within a circle of 1 arcmin with magnitude lower than 20 (with the first two factors), and the third factor will reduce this probability to reflect the fact that the QSOs with magnitudes 15 and 16 are much brighter (improbable) than those with magnitudes 19-20. As another example, imagine three QSOs of roughly the same magnitude 20 with distances to the galaxy of $0.1,0.2$ and 3.0 arcmin respectively. We will calculate the Poissonian probability of having background QSOs within a circle of 3 arcmin with magnitude 20 (with the first two factors), and the third factor will reduce this probability to reflect that the QSOs with distances 0.1 and 0.2 arcmin are much closer than expected randomly.

Table 1 gives $\lambda_{X, j}$ and $\lambda_{B, j}$ for each source, and the global probabilities $P_{X}$ and $P_{B}$ for each galaxy. The X-ray fluxes and the $B$-band magnitudes are listed in this table. If there is an optical identification, this is indicated in Col. 9.

\section{Results}

\subsection{Overall contamination}

A rough calculation with X-ray probabilities will give the total number of background sources:

number of background sources $\approx \sum_{j=1}^{99}\left(1-\mathrm{e}^{-13.4 \lambda_{X, i}}\right)=50.3$,

where the factor $13.4=766 / 57$ is the ratio of the total number of galaxies to those with at least one candidate ULX. The total number of background sources that we obtain with this expression is $51 \%$ of the background sources. It would be $>51.2$ ( $>52 \%$, only QSOs) if we performed the calculation with $\lambda_{B, i}$ instead of $\lambda_{X, i}$.

So far, 54 sources have been identified if we include the diffuse objects as identified (see Table 1$)$, and 36 of them $(67 \pm 11 \%)$ turned out to be contamination; 18 of the ULX candidates are sources belonging to the parent galaxy. This is slightly higher than our statistical estimate in X-ray. There may be a bias in the identified sources. They are on average brighter in the visible than the unobserved sources, although in principle fainter objects should have an even higher probability in the visible of being contamination. Other selection effects such as the avoidance of crowded regions in the spiral arms near the centre of the galaxies, where many optical counterparts are possible, could reduce this probability.

Ptak \& Colbert (2004) calculated a similar contamination: around $44 \%$. Liu et al. (2006) calculated a contamination ratio of $30 \%$ within $2 R_{25}$. Swartz et al. (2004) conclude that $\sim 25 \%$ of the sources ULXs candidates from Chandra ACIS may be background objects including $14 \%$ of the ULX candidates in the sample of spiral galaxies and $44 \%$ of those in elliptical galaxies, but their numbers are not directly comparable with those of the C02 sample because in some cases the Chandra fields do not cover the circles within $2 R_{25}$. For Irwin et al. (2004) the contamination is nearly total for elliptical galaxies and for the sources far from the centre in spiral galaxies. As said in the introduction, other authors (Kilgard et al. 2002; Schwartz et al. 2004;
Liu et al. 2006) also predicted a lower contribution of background sources for late-type galaxies.

\subsection{Contamination in each galaxy}

Table 1 presents the results of the statistical analysis. The expected mean number of random sources within the angular area is given in Cols. 4 and 7 for the analyses in the X-ray and in the optical respectively. The probabilities that all the ULXs detected in a given galaxy are contaminants are given in Cols. 5 and 8 . Since $\mathrm{C} 02 \mathrm{c}$ had a sample of 766 galaxies from which there are 57 galaxies with at least one candidate ULX, only galaxies with $P_{\mathrm{X}}$ or $P_{B}$ lower than $7 \times 10^{-5}$ will have at least one X-ray source within the $95 \%$ C.L. belonging to the galaxy, instead of all of them being background sources. Of the 57 galaxies, only 18 of them meet this criterion: NGC 1073, NGC 1313, NGC 1399, NGC 1672, Holmberg II, NGC 3628, NGC 4088, NGC 4319, NGC 4374, NGC 4472, NGC 4485, NGC 4559, NGC 4631, NGC 4649, NGC 4861, NGC 5128, NGC 5204 and NGC 5194. The average Hubble type of these galaxies is +3.2. We found some ellipticals (Hubble type: -5 or -4 ) with a low probability of all their ULXs being background sources, although no ULX was confirmed spectroscopically in them. See Sect. 6 for a discussion on their low probabilities of being background sources.

The second group with the remaining 39 galaxies would have 53 candidate ULXs, which mostly would be background sources: either QSOs or another X-ray emitter. The average Hubble type of the galaxies of this second group is +2.0. Indeed 22 sources of this second group already have a spectroscopic identification and 19 of them are QSOs or emission line galaxies with redshifts different from that of the main galaxy (see Table 1). Another source is a foreground $\mathrm{M}$ star. Moreover, if one looks at the photometry of the fourth release of the SDSS (Adelman-McCarthy et al. 2006), there are optical counterparts of seven IXOs of this second group within $10^{\prime \prime}(4,44,48$, 63, 64, 74 and 83) and three of them (4, 44 and 74) have colours typical of QSOs (according to the criterion in Eq. (1) of López-Corredoira \& Gutiérrez (2004), which means that they have a probability of being real QSOs of approximately 2/3).

This statistical analysis implies that, if one looks for ULXs, one should preferably observe the candidate ULXs of the 18 galaxies from the first group (with $P_{\mathrm{X}}$ or $P_{B}$ lower than $7 \times 10^{-5}$ ). For many of these 18 galaxies, spectroscopic identification is also available: there are confirmed sources belonging to the main galaxy for IXOs $5,7,8,31,65,68,72,73$, $77,80,81$ (see Table 1). Some other candidate ULXs are known to be bright diffuse sources that cannot be background QSOs: IXOs 26, 27, 42, 62, 79. There are also some identified QSOs with redshifts different from those of the parent galaxies: IXOs 19, 20, 57, 58, 69, 71; A3, 5, 9, 10, 13, 14, 21, 22. IXO75 is a foreground local M star; therefore, there still remain to be identified 15 IXOs of the first group: IXOs 17, 18, 25, 39, $50,51,55,56,59,60,61,66,70,76,78$. The variable X-ray source IXO 76 has a possible optical counterpart corresponding to a late $\mathrm{O}$ or early B star with magnitude 24.1 in the $F 555 \mathrm{~W}$ filter (Ghosh et al. 2006a), but this has not been confirmed spectroscopically. The fourth release of the SDSS (Adelman-McCarthy et al. 2006) has optical counterparts of IXOs 50, 51, 55, 56, 59, 60, 61 and 70, among which IXOs 50, 51, 55, 59, 70 have colours typical of QSOs (according to the criterion in Eq. (1) of López-Corredoira \& Gutiérrez (2004), although they might also be HII regions with the same colours), while IXOs 56, 60, 
61 have a different colour, so they should be ULXs, foreground stars, or emission line galaxies.

\subsection{Statistics with the clean sample}

We define the clean sample as the objects that are confirmed ULXs or candidates with low probabilities of being contaminants.

According to the discussion in the previous subsection, the objects that have not yet been observed spectroscopically and that have higher probabilities of being ULXs in the $\mathrm{C} 02$ catalogue are IXOs 17, 18, 25, 39, 66, 76 and 78. We removed IXOs 50, 51, 55, 59 and 70 from the initial list because of their typical QSO colours without being diffuse nebulae. We also removed IXOs 56, 60, 61 because their $\lambda_{B, j}>1$ while the $P_{B}$ and $P_{\mathrm{X}}$ of the parent galaxy NGC 4472 are not so low as to forbid a projection of seven QSOs (or any other kind of contaminating object).

The objects that are spectroscopically confirmed ULXs are (see Table 1) IXOs 5, 7, 8, 22, 31, 34, 65, 68, 72, 73, 77, 80, 81 as well as IXOs $26,27,42,62,79$, which were not observed spectroscopically but are bright diffuse sources, so they cannot be background QSOs, and are most probably HII regions of the parent galaxies.

In total, there are 25 objects. This sample might not be totally clean; even the objects identified as HII of the parent galaxy might correspond to wrong optical counterparts, but at least the contamination will be much lower than the original $\mathrm{C} 02$ sample. We will repeat the statistics of Ptak \& Colbert (2004) on the $\mathrm{C} 02$ sample, but only with these 25 objects. A histogram of energy distribution is shown in Fig. 1, and a histogram of the distribution according to Hubble type is presented in Fig. 2.

The distribution of energy peaks at intermediate energies $\left(\sim 4 \times 10^{39} \mathrm{erg} / \mathrm{s}\right)$, or it could be flat for lower energies (the Poisson errors do not allow a distinction). The distribution of the Hubble types peaks around late-type spirals, as expected; however, we have still two ellipticals. An examination of the IXOs from these ellipticals shows that they come from the unobserved candidates around the elliptical galaxies NGC 1399 and NGC 5128. These candidate ULXs may be background QSOs, but in such a case, we would have the problem of an anomalously high concentration of QSOs around NGC 1399 (see Sect. 6) and an inexplicably low value of $P_{X}$ in NGC 5128 due to contamination. NGC 1399 is a cD galaxy at the centre of a cluster; perhaps the ULXs near NGC 1399 belong to nearby galaxies in the cluster. The three ULXs in these two galaxies (IXOs 17, 18, 76) have not yet been observed; they are very faint (no optical counterpart has yet been found), so we cannot confirm whether the expectation by Irwin et al. (2004) that there are no ULXs associated with ellipticals is correct or not.

\section{QSOs with low probability of being background sources}

ULXs around some galaxies have been proposed to be QSOs or BL Lac objects with intrinsic redshift being ejected from a parent galaxy rather than black holes with masses $10^{2}-10^{4} M_{\odot}$ (Burbidge et al. 2003). It is clear that this cannot be the case for all candidate ULXs because there are many whose spectroscopy is different. We have tested whether the Burbidge et al. hypothesis could be applied to some of the objects. We perform a statistical analysis for each individual galaxy of the first group

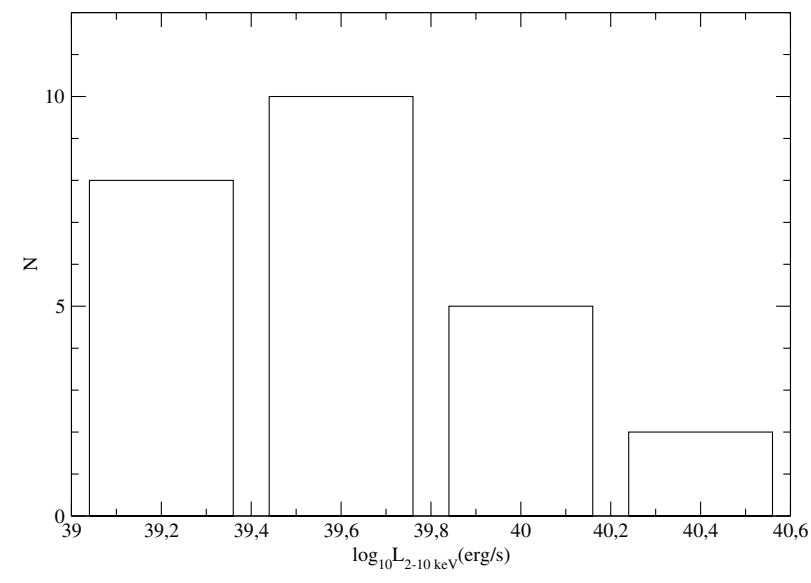

Fig. 1. Distribution of luminosities in the clean sample of ULXs.

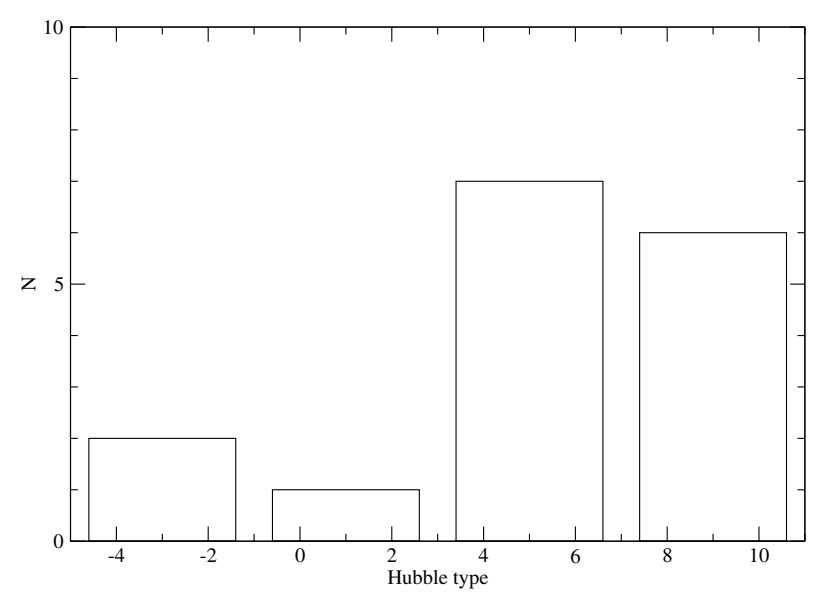

Fig. 2. Distribution of Hubble types of the galaxies with at least one member of the clean sample of ULXs.

(with $P_{\mathrm{X}}$ or $P_{B}$ lower than $7 \times 10^{-5}$ ) in which some QSO has been observed:

NGC 1073: with A3,5 as QSOs. Since IXO 5 is not a QSO, the probability of being a background object is not low for only two QSOs (excluding IXO 5 from the list): $P_{\mathrm{X}}=10^{-3}, P_{B}=10^{-4}$; quite possibly background sources. Note, however, that there is a third QSO around NGC 1073 which was not included here because the X-ray flux is less than the $\mathrm{C} 02$ requirements (equivalent to a luminosity $L_{\mathrm{X}}[2-10 \mathrm{keV}] \geq 10^{39} \mathrm{erg} / \mathrm{s}$ if they were at the distance of the galaxy). With this third QSO, the probability becomes lower, as claimed by Arp \& Sulentic (1979).

NGC 1399: with IXOs 19, 20 as QSOs. Were IXOs 17, 18 also QSOs, the probability of their being background sources would be very low; however, their optical spectra have still not been obtained, and they will probably not be observed in the near future because they are quite faint (they have no optical counterpart in the Palomar plates). For the two discovered QSOs (excluding IXOs 17, 18 from the list) the probabilities are: $P_{\mathrm{X}}=3 \times 10^{-3}, P_{B}=10^{-5}$. We suspect that there may be an error in the magnitudes given by NOMAD for the optical counterpart of IXO $20\left(m_{B}=15.2, m_{R}=18.1\right)$. 
NGC 3628: with A9,10,13,14 as QSOs. Again, the most intriguing case is IXO 39, with lower values of $\lambda$, but this has not been observed spectroscopically yet since its optical counterpart has not been detected. With the remaining four QSOs (excluding IXO 39 from the list): $P_{\mathrm{X}}=2 \times 10^{-5}, P_{B}=8 \times 10^{-5}$. There are still another three QSOs in A04 which do not follow the constraint $L_{X}[2-10 \mathrm{keV}] \geq 10^{39}$ but which, if included, will considerably reduce the probability by three orders of magnitude. Because of that, it is a known anomalous redshift candidate (Arp 2002), in which there is not only the overdensity but also an alignment of QSOs across the nucleus of NGC 3628 and their connection with $\mathrm{X}$-ray isophotes and coincidences in the alignment with some other filaments in the radio and optical wavelengths.

NGC 4319: with A21 as a QSO. This known QSO, Mrk 205, is a well known controversial case in the debate about anomalous redshifts. It is not only the low probability of finding so bright a QSO near NGC 4319 $\left(P_{\mathrm{X}}=4 \times 10^{-6}\right.$ and $\left.P_{B}=10^{-6}\right)$ but also the fact that it is connected by a small bridge to the galaxy (Sulentic \& Arp 1987a,b). There is also an almost continuous luminous connection extending from Mrk 205 into the nucleus of the spiral, and a corresponding feature on the opposite side of the disc appearing to link a bright UV knot $\left(m_{B}=19.5\right.$; with redshift of the galaxy, Sulentic \& Arp 1987b) with the nucleus (Sulentic \& Arp 1987a).

NGC 4374: with A22 as QSO. The probabilities for this object (excluding IXOs 50 and 51 from the list) are: $P_{\mathrm{X}}=2 \times 10^{-2}, P_{B}=3 \times 10^{-3}$, high enough for A22 most likely to be a background object. IXOs 50 and 51 have not yet been observed. There is no optical counterpart in the Palomar plates; however there are optical counterparts in SDSS-4th release survey: $u=24.63, g=25.11, r=24.80$ for IXO 50 (with a probable large error in these magnitudes because we are at the limit of the detection) and $u=20.97, g=20.70, r=20.01$ for IXO 51. Both of them might be QSOs according to their colour (see Eq. (1) of López-Corredoira $\&$ Gutiérrez 2004). Were these two cases really QSOs, we would have a very interesting new case of anomalous redshift.

NGC 4472: with IXOs 57, 58 as QSOs. And there remain IXOs 55, 56, 59, 60 and 61 to be identified. Only with the IXOs 57 and 58 as QSOs (excluding the other five IXOs from the list), the probabilities that they are both background objects are: $P_{\mathrm{X}}=0.1$, $P_{B}=2 \times 10^{-3}$. They are most probably two background QSOs. IXOs $55(u=20.57, g=20.66$, $r=20.41)$ and $59(u=20.68, g=20.07$, $r=20.08$ ) have SDSS colours of QSOs (see Eq. (1) of López-Corredoira \& Gutiérrez 2004), while IXOs $56(u=21.53, g=20.71, r=20.59)$, $60(u=22.89, g=21.36, r=20.81)$, and 61 $(u=23.42, g=22.22, r=21.35)$ are redder. Even where we have four QSOs (IXOs 55, 57, 58 and 59), the probability would be high enough to be a chance case of background sources.

NGC 4649: with IXOs 69 and 71 as QSOs. IXO 70 remains to be identified, although according to its SDSS colour
( $u=19.34, g=19.32, r=19.09)$, it might also be a QSO. Only with the IXOs 69 as 71 as QSOs (excluding IXO 70 from the list), the probabilities that they are both background objects are: $P_{\mathrm{X}}=$ $10^{-2}, P_{B}=2 \times 10^{-3}$. They are most probably two background QSOs.

Therefore, the possibly controversial cases with regard to anomalous redshift are only those that were known in the past, such as NGC 3628 and NGC 4319; the C02 catalog does not contain clear cases of QSOs with different redshifts and low probabilities of being background objects.

Radecke (1997) points out a detection of correlation between nearby Seyfert galaxies and X-ray sources, but the selection is different from those used in the $\mathrm{C} 02$ catalogue. Radecke uses only Seyfert galaxies while we use all kinds of galaxies. Radecke obtained the maximum correlation for very bright sources with $B$ magnitude lower than 10.5 , while most galaxies in the $\mathrm{C} 02$ sample are fainter. Radecke places the constraint on X-ray sources having more than 5 counts/ks while in the $\mathrm{C} 02$ sample most of the sources have less than 5 counts/ks. Therefore, there is no inconsitency between Radecke's analysis (which finds a number of X-ray sources around his galaxies that is considerably higher than expected from background sources; in the best case, he obtain an excess of over $250 \%$ ) and our result (an excess of $\sim 100 \%$ over the background sources, due to ULXs belonging to the galaxies but not being QSOs). It is also possible that part or all of the excess of sources around Seyfert galaxies in Radecke's analysis is due to the existence of a considerable number of ULXs belonging to the galaxies, these ULXs having the same redshift as the galaxies, so they could not be used as a claim of anomalous redshift. However, this hypothesis has not been tested yet.

\section{Conclusions}

From the above analysis we conclude the following:

- More than half of the ULX candidates in the C02c catalogue are background QSOs. This result, from the statistical analysis, is confirmed by follow-up spectroscopy of some of the sources.

- The clean sample is as follows. The spectroscopically confirmed ULXs are IXOs 5, 7, 8, 22, 31, 34, 65, 68, 72, 73, $77,80,81$, plus IXOs $26,27,42,62,79$, which were not observed spectroscopically but are bright diffuse sources, so they cannot be background QSOs and are most probably HII regions of the parent galaxies. Other objects that have not yet been observed spectroscopically and that have higher probabilities of being ULXs in the $\mathrm{C} 02$ catalogue are IXOs $17,18,25,39,66,76$ and 78 .

- Among the 87 sources in the C02 catalogue, there are no significant statistical cases that indicate non-cosmological redshifts in some QSOs associated with galaxies. Nonetheless, the problem of the anomalous redshifts is still open because there remain other known cases, for instance those of NGC 4319 or NGC 3628, with significantly low probabilities of their QSOs being background objects.

Acknowledgements. Thanks are given to the referee, P. Kaaret, for helpful comments. The authors were supported by the Ramón y Cajal Programme of the Spanish Science Ministry. Thanks are given to T. J. Mahoney (IAC) for the proofreading of the paper. 


\section{References}

Adelman-McCarthy, J. K., Agüeros, M. A., Allam, S. S., et al. 2006, ApJS, 162, 38

Arp, H. C. 1977, ApJ, 218, 72

Arp, H. C. 2002, A\&A, 391, 833

Arp, H. C., \& Sulentic, J. W. 1979, ApJ 229, 496

Arp, H. C., Gutiérrez, C. M., \& López-Corredoira, M. 2004, A\&A, 418, 877 (A04)

Boyle, B. J., Shanks, T., Croom, S. M., et al. 2000, MNRAS, 317, 1014

Burbidge, G., Burbidge, E. M., \& Arp, H. 2003, A\&A, 400, L17

Cheney, J. E., \& Rowan-Robinson, M. 1981, MNRAS, 195, 497

Clark, D. M., Christopher, M. H., Eikenberry, S. S., et al. 2005, ApJ, 631, L109

Colbert, E. J. M., \& Mushotzky, R. F. 1999, ApJ, 519, 89

Colbert, E. J. M., \& Ptak, A. F. 2002, ApJS, 143, 25 (C02)

Gao, Y., Wang, Q. D., Appleton, P. N., \& Lucas, R. A. 2003, ApJ, 596, L171

Ghosh, K. K., Finger, M. K., Swartz, D. A., Tennannt, A. F., \& Wu, K. 2006a, ApJ, 640, 459

Ghosh, K. K., Swartz, D. A., Tennant, A. F., et al. 2006b, ApJ, submitted Gutiérrez, C. M. 2006, ApJ, 640, L17

Gutiérrez, C. M., \& López-Corredoira, M. 2005, ApJ, 622, L89

Gutiérrez, C. M., \& López-Corredoira, M. 2006, in preparation

Hao, L., Strauss, M. A., Fan, X., et al. 2005, AJ, 129, 1795

Hasinger, G. 1998, Astron. Nachr., 319, 37

Hasinger, G., Burg, R., Giacconi, R., et al. 1993, A\&A, 275,

Irwin, J. A., Bregman, J. N., \& Athey, A. E. 2004, ApJ, 601, L143

Kaaret, P. 2005, ApJ, 629, 233

Kaaret, P., Ward, M.-J., \& Zezas, A. 2004, MNRAS, 351, L83

Kilgard, R. E., Kaaret, P., Krauss, M. I., et al. 2002, ApJ, 573, 138

Larsen, J. A., \& Humphreys, R. M. 2003, AJ, 125, 1958

Lehmann, I., Becker, T., Fabrika, S., et al. 2005, A\&A, 431, 847

Lira, P., Lawrence, A., \& Johnson, R. A. 2000, MNRAS, 319, 17
Liu, J.-F., \& Bregman, J.-N. 2005, ApJS, 157, 59

Liu, J.-F., Bregman, J.-N., \& Seitzer, P. 2004, ApJ, 602, 249

Liu, J.-F., Bregman, J.-N., Seitzer, P., \& Irwin, J. 2005 [arXiv: astro-ph/0501310]

Liu, J.-F., Bregman, J. N., \& Irwin, J. 2006, ApJ, 642, 171

Liu, Q.-Z., \& Mirabel, I. F. 2005, A\&A, 429, 1125

López-Corredoira, M., \& Gutiérrez, C. M. 2004, A\&A, 421, 407

Makishima, K., Kubota, A., Mizuno, T., et al. 2000, ApJ, 535, 632

Meyer, M. J., Drinkwater, M. J., Phillips, S., \& Couch, W. J. 2001, MNRAS, 324,343

Mucciarelli, P., Zampieri, L., Falomo, R., Turolla, R., \& Treves, A. 2005, ApJ, 633, L101

Pakull, M. W., \& Mirioni, L. 2002, in New Visions of the X-ray Universe in the XMM-Newton and Chandra Era, ESTEC, The Netherlands, preprint [arXiv: astro-ph/0202488]

Ptak, A., \& Colbert, E. 2004, ApJ, 606, 291

Radecke, H. D. 1997, A\&A, 319, 18

Ramsey, C. J., Williams, R. M., Gruendl, R. A., et al. 2006, ApJ, 641, 241

Robin, A. C., Reylé, C., Derrière, S., \& Picaud, S. 2003, A\&A, 409, 523

Smith, B. J., Struck, C., \& Nowak, M. A. 2005, AJ, 129, 1350

Sulentic, J. W., \& Arp, H. C. 1987a, ApJ, 319, 687

Sulentic, J. W., \& Arp, H. C. 1987b, ApJ, 319, 693

Swartz, D., Ghosh, K., Tennant, A., \& Wu, K. 2004, ApJS, 154, 519

Voges, W., et al. 1999, in Diffuse thermal and relativistic plasma in galaxy clusters, ed. H. Bohringer, L. Feretti, \& P. Schuecker (Garching: Max-PlanckInstitut für Extraterrestrische Physik), 179

Wong, D. S., Chornock, R., \& Filippenko, A. V. 2006, Populations of High Energy Sources in Galaxies, Proc. IAU Symp. 230, ed. E. J. A. Meurs, \& G. Fabbiano (Cambridge: Cambridge University Press), 306

Zampieri, L., Mucciarelli, P., Falomo, R., et al. 2004, ApJ, 603, 523

Zickgraf, F.-J., Engels, D., Hagen, H.-J., Reimers, D., \& Voges, W. 2003, A\&A, 406,535 